PROCEEDINGS OF THE

AMERICAN MATHEMATICAL SOCIETY

Volume 140, Number 11, November 2012, Pages 4003-4010

S 0002-9939(2012)11206-7

Article electronically published on February 28, 2012

\title{
PLURISUBHARMONIC FUNCTIONS AND NEF CLASSES ON COMPLEX MANIFOLDS
}

\author{
VALENTINO TOSATTI AND BEN WEINKOVE
}

(Communicated by Jianguo Cao)

\begin{abstract}
We prove the existence of plurisubharmonic functions with prescribed logarithmic singularities on complex 3-folds equipped with a nef class of positive volume. We prove the same result for rational classes on Moishezon $n$-folds.
\end{abstract}

\section{INTRODUCTION}

We recall that a function $f: \Omega \rightarrow[-\infty, \infty)$ for a domain $\Omega \subset \mathbb{C}^{n}$ is plurisubharmonic if it is upper semi-continuous and for every $a, b \in \mathbb{C}^{n}$, the map

$$
z \in \mathbb{C} \mapsto f(a+b z) \in[-\infty, \infty)
$$

is subharmonic where it is defined.

Now let $(X, \omega)$ be a compact Kähler manifold of complex dimension $n$. There are complex coordinate charts $B_{i}$ on which we can write $\omega=\frac{\sqrt{-1}}{\pi} \partial \bar{\partial} g_{i}$ for smooth potential functions $g_{i}$ (unique up to adding pluriharmonic functions). We define a function $\varphi: X \rightarrow[-\infty,+\infty)$ to be $\omega$-plurisubharmonic if $\varphi$ is upper semicontinuous, not identically $-\infty$, and each $g_{i}+\varphi$ is plurisubharmonic (clearly, this does not depend on the choice of potentials $g_{i}$ ). A basic fact is that an $L^{1}$ function $\varphi$ on $X$ satisfying

$$
\omega+\frac{\sqrt{-1}}{\pi} \partial \bar{\partial} \varphi \geqslant 0
$$

in the sense of currents, agrees with a unique $\omega$-plurisubharmonic function almost everywhere. Conversely, every $\omega$-plurisubharmonic $\varphi$ is in $L^{1}$ and satisfies (1.1).

The space of $\omega$-plurisubharmonic $\varphi$ has been the focus of considerable study in the last few decades. Note that every closed positive real $(1,1)$-current cohomologous to $[\omega] \in H^{1,1}(X, \mathbb{R})$ can be written as $\omega+\frac{\sqrt{-1}}{\pi} \partial \bar{\partial} \varphi$ for some such $\varphi$.

It was shown in [18, 26] (see also [27]) that there exists a constant $\alpha>0$ depending only on $[\omega]$ such that

$$
\int_{X} e^{-\alpha \varphi} \omega^{n} \leqslant C
$$

for all $\omega$-plurisubharmonic $\varphi$. This shows in particular that singularities of $\varphi$ can be at most logarithmic.

Received by the editors March 29, 2011 and, in revised form, April 19, 2011.

2010 Mathematics Subject Classification. Primary 53C55; Secondary 32W20, 32U05, 32 U25.

Research supported in part by National Science Foundation grants DMS-08-48193 and DMS10-05457.

The second author was also supported in part by a Sloan Foundation fellowship.

(c) 2012 American Mathematical Society Reverts to public domain 28 years from publication 
An interesting and well-known problem is: can we find a $\varphi$ with prescribed logarithmic singularities at given points on $X$ ? In the case when $[\omega]$ is the Chern class of a holomorphic line bundle $L$, this is equivalent to prescribing singular Hermitian metrics on $L$. This can be naturally extended to line bundles $L$ which are only nef. Deep results in [6, 1, 25] and others used the construction of singular $\varphi$ to prove effective results in algebraic geometry.

In this short paper we investigate this problem on general complex (non-Kähler) manifolds. Our motivation is to try to understand whether techniques from Kähler geometry can be extended to non-Kähler complex geometry, at least when natural analogues exist.

Suppose now that $X$ is only a compact complex manifold. Let $\beta$ be a closed real $(1,1)$-form on $X$, consider the (finite-dimensional) real Bott-Chern cohomology group

$$
H_{\mathrm{BC}}^{1,1}(X, \mathbb{R})=\frac{\{\text { closed real }(1,1) \text {-forms }\}}{\left\{\frac{\sqrt{-1}}{\pi} \partial \bar{\partial} \psi, \psi \in C^{\infty}(X, \mathbb{R})\right\}},
$$

and call $[\beta]$ the class of $\beta$ in $H_{\mathrm{BC}}^{1,1}(X, \mathbb{R})$.

Since a positive $\beta$ only exists if $X$ is Kähler, we consider instead the case when the class $[\beta]$ is nef (as defined in [9]), which means that for any $\varepsilon>0$ there exists a representative $\beta+\frac{\sqrt{-1}}{\pi} \partial \bar{\partial} \psi_{\varepsilon}$ so that $\beta+\frac{\sqrt{-1}}{\pi} \partial \bar{\partial} \psi_{\varepsilon}>-\varepsilon \omega$, where $\omega$ is some fixed Hermitian metric on $X$.

The closed form $\beta$ admits local potential functions, and thus we can define the notion of $\beta$-plurisubharmonic in the same way as described above. Under the cohomological assumption that $\int_{X} \beta^{n}>0$, we look for $\beta$-plurisubharmonic functions $\varphi$ with prescribed logarithmic singularities.

Our main result is the following:

Main Theorem. Let $X$ be a compact complex manifold of dimension $n$. Suppose there exists a class $[\beta] \in H_{\mathrm{BC}}^{1,1}(X, \mathbb{R})$ which is nef and satisfies $\int_{X} \beta^{n}>0$. Assume either

(i) $n=2$ or $n=3$

or

(ii) $X$ is Moishezon and $[\beta] \in H_{\mathrm{BC}}^{1,1}(X, \mathbb{Q}):=H_{\mathrm{BC}}^{1,1}(X, \mathbb{R}) \cap H^{2}(X, \mathbb{Q})$.

Fix points $x_{1}, \ldots, x_{N} \in X$ and choose positive real numbers $\tau_{1}, \ldots, \tau_{N}$ so that

$$
\sum_{j} \tau_{j}^{n}<\int_{X} \beta^{n} .
$$

Then there exists a $\beta$-plurisubharmonic $\varphi$ with logarithmic poles at $x_{1}, \ldots, x_{N}$ :

$$
\varphi(z) \leqslant \tau_{j} \log |z|+O(1),
$$

in a coordinate neighborhood $\left(z_{1}, \ldots, z_{n}\right)$ centered at $x_{j}$, where $|z|^{2}=\left|z_{1}\right|^{2}+\cdots+$ $\left|z_{n}\right|^{2}$. In particular, the Lelong number of $\varphi$ at each point $x_{j}$ is at least $\tau_{j}$.

Recall that a Moishezon manifold is a compact complex manifold which is bimeromorphic to a projective manifold. An equivalent definition is that a Moishezon manifold is a compact complex manifold admitting a big line bundle $L$ (meaning $\operatorname{dim} H^{0}\left(X, L^{k}\right)>c k^{n}$ for $k$ large, for some fixed $\left.c>0\right)$.

If $X$ is Kähler (without imposing (i) or (ii)), this result is due to Demailly [], whose proof made use of Yau's solution of the complex Monge-Ampère equation on Kähler manifolds [30]. 
In the case of one point $(N=1)$, inequality (1.2) is sharp in general: taking $X=\mathbb{C P}^{n}$ and $[\beta]$ to be the (ample) anticanonical class (so $\beta$ is $n+1$ times the Fubini-Study metric), equation (1.2) says that $\tau<n+1$, and it is well known (see e.g. 4, Proposition 2.1]) that $n+1$ is indeed the maximum order of a logarithmic pole of any $\beta$-plurisubharmonic function.

We remark that our result in case (i) is only really new in the case $n=3$. The reason is that in dimension 2, any surface $X$ as in the Main Theorem is necessarily Kähler. In fact, the existence of a closed real $(1,1)$-form $\beta$ with $\int_{X} \beta^{2}>0$ implies that the intersection form on $H^{1,1}(X, \mathbb{R})$ is not negative definite, and thanks to a classical theorem of Kodaira [20] this implies that $b_{1}(X)$ is even. A theorem of Miyaoka and Siu 23, 24, (see also [2, 21]) then implies that $X$ is Kähler.

On the other hand there are certainly many non-Kähler 3-folds satisfying the hypotheses of the Main Theorem (see for example [17] and the description in [16, Example 3.4.1, p.443]). Indeed, such manifolds were discussed by Demailly and Păun in [9], where it was conjectured that a compact complex $n$-fold $X$ with a nef class $[\beta]$ of type $(1,1)$ with $\int_{X} \beta^{n}>0$ is bimeromorphic to a Kähler manifold.

Demailly [6] proved the Kähler version of the Main Theorem using Yau's existence result for solutions to the complex Monge-Ampère equation on Kähler manifolds [30. In this paper we follow along the same lines of argument as Demailly, but now apply the recent extension of Yau's Theorem to general complex manifolds 28] (see also [3, 14, 29, 11, 1, 13). However, a difficulty arises here in the non-Kähler case due to the fact that for a $(1,1)$-form $\Omega$ and a function $f$, the equality

$$
\int_{X}\left(\Omega+\frac{\sqrt{-1}}{\pi} \partial \bar{\partial} f\right)^{n}=\int_{X} \Omega^{n}
$$

does not hold in general if $\Omega$ is not closed. We can overcome this obstacle in dimensions 2 and 3 by making use of Gauduchon metrics.

We conjecture that the Main Theorem holds for any dimension without the Moishezon and rationality assumptions in (ii).

\section{Proof of the Main Theorem}

Let $\omega$ be a Gauduchon metric on $X$, which means that $\partial \bar{\partial}\left(\omega^{n-1}\right)=0$ (such a metric always exists [12]). Following Demailly [6], we choose coordinates $z^{1}, \ldots, z^{n}$ in a neighborhood centered at $x_{j}$ and put

$$
\gamma_{j, \varepsilon}=\frac{\sqrt{-1}}{\pi} \partial \bar{\partial}\left(\chi\left(\log \frac{|z|}{\varepsilon}\right)\right),
$$

where $|z|^{2}=\left|z^{1}\right|^{2}+\cdots+\left|z^{n}\right|^{2}$, and $\chi: \mathbb{R} \rightarrow \mathbb{R}$ is smooth, convex, increasing and satisfies $\chi(t)=t$ for $t \geqslant 0$ and $\chi(t)=-1 / 2$ for $t \leqslant-1$. Observe that $\gamma_{j, \varepsilon}$ is a nonnegative real $(1,1)$-form defined only on this coordinate chart, which equals $\frac{\sqrt{-1}}{\pi} \partial \bar{\partial} \log |z|$ outside the ball $\{|z| \leqslant \varepsilon\}$. We therefore have that $\gamma_{j, \varepsilon}^{n}=0$ if $|z|>\varepsilon$, so we can extend it to zero on the whole of $X$. This way, $\gamma_{j, \varepsilon}^{n}$ is a smooth nonnegative $(n, n)$-form on $X$ that satisfies

$$
\int_{X} \gamma_{j, \varepsilon}^{n}=\int_{|z| \leqslant \varepsilon} \gamma_{j, \varepsilon}^{n}=\int_{|z| \leqslant \varepsilon}\left(\frac{\sqrt{-1}}{\pi} \partial \bar{\partial} \log |z|\right)^{n}=1,
$$

using Stokes' theorem, and $\gamma_{j, \varepsilon}^{n} \rightarrow \delta_{x_{j}}$ as $\varepsilon \rightarrow 0$. Since $\beta$ is nef, for any $\varepsilon>0$ there exists a smooth function $\psi_{\varepsilon}$ such that $\beta+\varepsilon \omega+\frac{\sqrt{-1}}{\pi} \partial \bar{\partial} \psi_{\varepsilon}$ is Hermitian. 
Now recall that the Hermitian version of Yau's theorem [28] states that given a Hermitian metric $\hat{\omega}$ and a smooth function $F$ there exists a unique smooth $f$ and a unique constant $K>0$ solving

$$
\left(\hat{\omega}+\frac{\sqrt{-1}}{\pi} \partial \bar{\partial} f\right)^{n}=K e^{F} \hat{\omega}^{n}, \quad \hat{\omega}+\frac{\sqrt{-1}}{\pi} \partial \bar{\partial} f>0, \quad \sup _{X} f=0 .
$$

Note that, in the case of Yau's theorem where $\hat{\omega}$ is Kähler, integration by parts shows that $K=\int_{X} \hat{\omega}^{n} / \int_{X} e^{F} \hat{\omega}^{n}$. In the non-Kähler case, no such formula holds in general and this is the source of the difficulty.

Applying this with reference metric $\beta+\varepsilon \omega+\frac{\sqrt{-1}}{\pi} \partial \bar{\partial} \psi_{\varepsilon}$, we obtain a smooth $\varphi_{\varepsilon}$ with

$$
\left(\beta+\varepsilon \omega+\frac{\sqrt{-1}}{\pi} \partial \bar{\partial}\left(\psi_{\varepsilon}+\varphi_{\varepsilon}\right)\right)^{n}=C_{\varepsilon}\left(\sum_{j} \tau_{j}^{n} \gamma_{j, \varepsilon}^{n}+\delta \omega^{n}\right)
$$

and

$$
\beta+\varepsilon \omega+\frac{\sqrt{-1}}{\pi} \partial \bar{\partial}\left(\psi_{\varepsilon}+\varphi_{\varepsilon}\right)>0, \quad \sup _{X}\left(\psi_{\varepsilon}+\varphi_{\varepsilon}\right)=0,
$$

where $\delta>0$ is fixed and $C_{\varepsilon}$ is a (uniquely determined) positive constant. The key fact that we need now is the lower bound $C_{\varepsilon} \geqslant 1$ for $\varepsilon$ and $\delta$ sufficiently small. We remark that there are some formal similarities between the argument given here for this lower bound and the proofs of Proposition 3.8 in [10] and Theorem 1.2 in [19.

We consider first the case (i) when $n=2$. Then $\partial \bar{\partial} \omega=0$ and, using the fact that $\beta$ is closed, we have

$$
\begin{aligned}
C_{\varepsilon} & =\frac{\int_{X}\left(\beta+\varepsilon \omega+\frac{\sqrt{-1}}{\pi} \partial \bar{\partial}\left(\psi_{\varepsilon}+\varphi_{\varepsilon}\right)\right)^{2}}{\int_{X} \sum_{j} \tau_{j}^{2} \gamma_{j, \varepsilon}^{2}+\delta \omega^{2}}=\frac{\int_{X}(\beta+\varepsilon \omega)^{2}}{\sum_{j} \tau_{j}^{2}+\delta \int_{X} \omega^{2}} \\
& \geqslant \frac{\int_{X} \beta^{2}-\varepsilon^{2} \int_{X} \omega^{2}}{\sum_{j} \tau_{j}^{2}+\delta \int_{X} \omega^{2}},
\end{aligned}
$$

since

$$
\int_{X}(\beta+\varepsilon \omega)^{2}=\int_{X} \beta^{2}+2 \varepsilon \int_{X}\left(\beta+\varepsilon \omega+\frac{\sqrt{-1}}{\pi} \partial \bar{\partial} \psi_{\varepsilon}\right) \wedge \omega-\varepsilon^{2} \int_{X} \omega^{2} .
$$

Choosing $\delta$ sufficiently small and using (1.2), we get for any $\varepsilon>0$ sufficiently small,

$$
C_{\varepsilon} \geqslant 1 \text {. }
$$

We now consider the case when $n=3$. For simplicity call $\beta_{\varepsilon}=\beta+\varepsilon \omega+$ $\frac{\sqrt{-1}}{\pi} \partial \bar{\partial}\left(\psi_{\varepsilon}+\varphi_{\varepsilon}\right)$ and note that

$$
\beta+\frac{\sqrt{-1}}{\pi} \partial \bar{\partial}\left(\psi_{\varepsilon}+\varphi_{\varepsilon}\right)=\beta_{\varepsilon}-\varepsilon \omega,
$$

and so

$$
\int_{X}\left(\beta_{\varepsilon}-\varepsilon \omega\right)^{3}=\int_{X}\left(\beta+\frac{\sqrt{-1}}{\pi} \partial \bar{\partial}\left(\psi_{\varepsilon}+\varphi_{\varepsilon}\right)\right)^{3}=\int_{X} \beta^{3}>0 .
$$

Using the Gauduchon condition $\partial \bar{\partial}\left(\omega^{2}\right)=0$, we see that

$$
\int_{X} \beta_{\varepsilon} \wedge \omega^{2}=\int_{X}(\beta+\varepsilon \omega) \wedge \omega^{2} \leqslant C \int_{X} \omega^{3} .
$$


On the other hand, using (2.5), we have

$$
\begin{aligned}
\int_{X} \beta_{\varepsilon}^{3} & =\int_{X}\left(\beta_{\varepsilon}-\varepsilon \omega+\varepsilon \omega\right)^{3} \\
& =\int_{X}\left(\beta_{\varepsilon}-\varepsilon \omega\right)^{3}+3 \varepsilon \int_{X} \beta_{\varepsilon}^{2} \wedge \omega-3 \varepsilon^{2} \int_{X} \beta_{\varepsilon} \wedge \omega^{2}+\varepsilon^{3} \int_{X} \omega^{3} \\
& \geqslant \int_{X} \beta^{3}-C^{\prime} \varepsilon^{2},
\end{aligned}
$$

and so

$$
C_{\varepsilon}=\frac{\int_{X} \beta_{\varepsilon}^{3}}{\int_{X} \sum_{j} \tau_{j}^{3} \gamma_{j, \varepsilon}^{3}+\delta \omega^{3}} \geqslant \frac{\int_{X} \beta^{3}-C^{\prime} \varepsilon^{2}}{\sum_{j} \tau_{j}^{3}+\delta \int_{X} \omega^{3}},
$$

and hence choosing $\delta$ sufficiently small and using (1.2), we get for $\varepsilon>0$ sufficiently small

$$
C_{\varepsilon} \geqslant 1
$$

Finally we prove $C_{\varepsilon} \geqslant 1$ in the case (ii). Because $[\beta]$ is rational we have $\ell[\beta]=$ $c_{1}(L)$ for some line bundle $L$ over $X$ and some integer $\ell \geqslant 1$. In this case there exists a Hermitian metric $h_{\varepsilon}$ on $L$ such that $\frac{1}{\ell} c_{1}\left(L, h_{\varepsilon}\right)=\beta+\frac{\sqrt{-1}}{\pi} \partial \bar{\partial}\left(\psi_{\varepsilon}+\varphi_{\varepsilon}\right)$, where $c_{1}\left(L, h_{\varepsilon}\right)$ is the curvature form of the Hermitian metric $h_{\varepsilon}$. Denote by $X(0)$ the set of $x \in X$ such that $c_{1}\left(L, h_{\varepsilon}\right)$ has 0 negative eigenvalues. We now apply Demailly's holomorphic Morse inequalities [5] to see that for $k$ large we have

$$
\begin{aligned}
\operatorname{dim} H^{0}\left(X, L^{k}\right) & \leqslant \frac{k^{n}}{n !} \int_{X(0)} c_{1}\left(L, h_{\varepsilon}\right)^{n}+o\left(k^{n}\right) \\
& \leqslant \frac{k^{n}}{n !} \int_{X(0)}\left(c_{1}\left(L, h_{\varepsilon}\right)+\varepsilon \ell \omega\right)^{n}+o\left(k^{n}\right) \\
& =\frac{C_{\varepsilon} \ell^{n} k^{n}}{n !} \int_{X(0)}\left(\sum_{j} \tau_{j}^{n} \gamma_{j, \varepsilon}^{n}+\delta \omega^{n}\right)+o\left(k^{n}\right) \\
& \leqslant \frac{C_{\varepsilon} \ell^{n} k^{n}}{n !}\left(\sum_{j} \tau_{j}^{n}+\delta \int_{X} \omega^{n}\right)+o\left(k^{n}\right) .
\end{aligned}
$$

We now estimate the number of sections of $L^{k}$ using the Riemann-Roch theorem. Since the manifold $X$ is Moishezon there exists a modification $\mu: \tilde{X} \rightarrow X$ with $\tilde{X}$ a projective manifold. The pullback $\mu^{*} L$ is then a nef line bundle on $\tilde{X}$ with $\int_{\tilde{X}} c_{1}\left(\mu^{*} L\right)^{n}=\ell^{n} \int_{X} \beta^{n}>0$. Because $\mu^{*} L$ is nef, its higher cohomology groups satisfy $\operatorname{dim} H^{q}\left(\tilde{X}, \mu^{*} L^{k}\right)=O\left(k^{n-1}\right)$, for $q>0$ (see Example 1.2.36 in [22]), and a standard Leray spectral sequence argument (see (2.1) in [8]) shows that $\operatorname{dim} H^{q}\left(X, L^{k}\right)=O\left(k^{n-1}\right)$, for $q>0$. By the Riemann-Roch theorem we now have

$$
\operatorname{dim} H^{0}\left(X, L^{k}\right)=\frac{\ell^{n} k^{n}}{n !} \int_{X} \beta^{n}+o\left(k^{n}\right) .
$$

Combining (2.6) and (2.7) and taking $k$ large we get

$$
\int_{X} \beta^{n} \leqslant C_{\varepsilon}\left(\sum_{j} \tau_{j}^{n}+\delta \int_{X} \omega^{n}\right) .
$$

Choosing $\delta>0$ sufficiently small we obtain $C_{\varepsilon} \geqslant 1$. 
We claim that $\psi_{\varepsilon}+\varphi_{\varepsilon}$ is uniformly bounded in $L^{1}$. This is because we can choose a large constant $A$ so that $A \omega \geqslant \beta+\varepsilon \omega$ for all $0<\varepsilon \leqslant 1$, and then the function $\psi_{\varepsilon}+\varphi_{\varepsilon}$ satisfies $A \omega+\frac{\sqrt{-1}}{\pi} \partial \bar{\partial}\left(\psi_{\varepsilon}+\varphi_{\varepsilon}\right)>0$, and then Proposition 2.1 in [11] (for example) gives a uniform $L^{1}$ bound that depends only on $X, A, \omega$.

This implies that there is a sequence $\varepsilon_{k} \rightarrow 0$ such that $\psi_{\varepsilon_{k}}+\varphi_{\varepsilon_{k}}$ converges in $L^{1}$ to a $\beta$-plurisubharmonic function $\varphi$. Indeed, we can recover this from the local statement about compactness of plurisubharmonic functions in a domain in $\mathbb{C}^{n}$ which are uniformly bounded in $L^{1}$, in the following way: we cover $X$ with finitely many coordinate charts $B_{i}$ so that on each $B_{i}$ there is a smooth function $\rho_{i}$ with $\frac{\sqrt{-1}}{\pi} \partial \bar{\partial} \rho_{i}>\beta+\omega$. Then on each $B_{i}$ the functions $\rho_{i}+\psi_{\varepsilon}+\varphi_{\varepsilon}$ are plurisubharmonic and uniformly bounded in $L^{1}$ (independent of $\varepsilon$ ), so the local statement applies.

We now show that $\varphi$ has the desired logarithmic singularities.

Take $\Omega$ to be a neighborhood of $x_{j}$ (which we can assume contains the set $\{|z|<1\})$ and consider the smooth plurisubharmonic function on $\Omega$,

$$
u=C_{\varepsilon}^{1 / n} \tau_{j}(\chi(\log (|z| / \varepsilon))+\log \varepsilon)+C_{1}
$$

for $C_{1}$ a large constant, where $\chi$ is the same cutoff function as in (2.4). Let $h$ be a smooth function on $\bar{\Omega}$ with

$$
\frac{\sqrt{-1}}{\pi} \partial \bar{\partial} h \geqslant \beta+\omega
$$

and put $v=h+\psi_{\varepsilon}+\varphi_{\varepsilon}$, so $v$ is a smooth plurisubharmonic function on $\Omega$ which is bounded from above by $C_{0}$, say.

Then if $\varepsilon$ is sufficiently small and $C_{1}$ is sufficiently large we have

$$
\left.u\right|_{\partial \Omega}=C_{\varepsilon}^{1 / n} \tau_{j} \log |z|+C_{1} \geqslant C_{0} \geqslant\left. v\right|_{\partial \Omega} .
$$

But in addition we have on $\Omega$

$$
\begin{aligned}
\left(\frac{\sqrt{-1}}{\pi} \partial \bar{\partial} v\right)^{n} & \geqslant\left(\beta+\varepsilon \omega+\frac{\sqrt{-1}}{\pi} \partial \bar{\partial}\left(\psi_{\varepsilon}+\varphi_{\varepsilon}\right)\right)^{n} \\
& \geqslant C_{\varepsilon} \tau_{j}^{n} \gamma_{j, \varepsilon}^{n}=\left(\frac{\sqrt{-1}}{\pi} \partial \bar{\partial} u\right)^{n} .
\end{aligned}
$$

Then by the Bedford-Taylor comparison principle for Monge-Ampère (e.g. Lemma 6.7 in [6]),

$$
u \geqslant v \text { on } \Omega,
$$

and hence when $|z|<1 / 2$ and $\varepsilon$ is small we have

$$
\begin{aligned}
\psi_{\varepsilon}+\varphi_{\varepsilon} & \leqslant C_{\varepsilon}^{1 / n} \tau_{j}(\chi(\log (|z| / \varepsilon))+\log \varepsilon)+C_{2} \leqslant C_{\varepsilon}^{1 / n} \tau_{j} \log (|z|+\varepsilon)+C_{2} \\
& \leqslant \tau_{j} \log (|z|+\varepsilon)+C_{2},
\end{aligned}
$$

where we are using the fact that $C_{\varepsilon} \geqslant 1$. Since $\psi_{\varepsilon}+\varphi_{\varepsilon}$ converges to $\varphi$ in $L^{1}$, Hartogs' Lemma (see for example Proposition 2.6 (2) in [15]) implies (1.3). Q.E.D.

Let us remark here that, in any dimension, the constant $C_{\varepsilon}$ is always bounded above (independent of $\varepsilon$ but depending on $\delta$ ) when $\varepsilon$ is small. Indeed, at the point 
on $X$ where the function $\psi_{\varepsilon}+\varphi_{\varepsilon}$ achieves its maximum, we have that $\beta+\varepsilon \omega$ is positive definite and moreover $\frac{\sqrt{-1}}{\pi} \partial \bar{\partial}\left(\psi_{\varepsilon}+\varphi_{\varepsilon}\right) \leqslant 0$, and so at that point we have

$$
\begin{aligned}
C \omega^{n} & \geqslant(\beta+\varepsilon \omega)^{n} \geqslant\left(\beta+\varepsilon \omega+\frac{\sqrt{-1}}{\pi} \partial \bar{\partial}\left(\psi_{\varepsilon}+\varphi_{\varepsilon}\right)\right)^{n} \\
& =C_{\varepsilon}\left(\sum_{j} \tau_{j}^{n} \gamma_{j, \varepsilon}^{n}+\delta \omega^{n}\right) \geqslant C_{\varepsilon} \delta \omega^{n},
\end{aligned}
$$

giving $C_{\varepsilon} \leqslant C / \delta$.

A similar argument only gives a lower bound of the form $C_{\varepsilon} \geqslant c \varepsilon^{3 n}$, in any dimension. In fact, a direct calculation shows that on $X$ we have

$$
\gamma_{j, \varepsilon}^{n} \leqslant \frac{C}{\varepsilon^{2 n}} \omega^{n}
$$

for a constant $C$ independent of $\varepsilon$. We then compute

$$
\left(\beta+\varepsilon \omega+\frac{\sqrt{-1}}{\pi} \partial \bar{\partial}\left(\psi_{\varepsilon}+\varphi_{\varepsilon}\right)\right)^{n}=C_{\varepsilon}\left(\sum_{j} \tau_{j}^{n} \gamma_{j, \varepsilon}^{n}+\delta \omega^{n}\right) \leqslant \frac{C}{\varepsilon^{2 n}} C_{\varepsilon} \omega^{n} .
$$

Recall that since $[\beta]$ is nef there is a smooth function $\psi_{\varepsilon / 2}$ so that $\beta+\frac{\varepsilon}{2} \omega+$ $\frac{\sqrt{-1}}{\pi} \partial \bar{\partial} \psi_{\varepsilon / 2}>0$. We then write

$$
\begin{aligned}
\beta+\varepsilon \omega+\frac{\sqrt{-1}}{\pi} \partial \bar{\partial}\left(\psi_{\varepsilon}+\varphi_{\varepsilon}\right)= & \left(\beta+\frac{\varepsilon}{2} \omega+\frac{\sqrt{-1}}{\pi} \partial \bar{\partial} \psi_{\varepsilon / 2}\right) \\
& +\left(\frac{\varepsilon}{2} \omega+\frac{\sqrt{-1}}{\pi} \partial \bar{\partial}\left(\psi_{\varepsilon}+\varphi_{\varepsilon}-\psi_{\varepsilon / 2}\right)\right) .
\end{aligned}
$$

At the point on $X$ where $\psi_{\varepsilon}+\varphi_{\varepsilon}-\psi_{\varepsilon / 2}$ achieves its minimum we have $\frac{\sqrt{-1}}{\pi} \partial \bar{\partial}\left(\psi_{\varepsilon}+\right.$ $\left.\varphi_{\varepsilon}-\psi_{\varepsilon / 2}\right) \geqslant 0$, and so at that point

$$
\frac{\varepsilon^{n}}{2^{n}} \omega^{n} \leqslant\left(\beta+\varepsilon \omega+\frac{\sqrt{-1}}{\pi} \partial \bar{\partial}\left(\psi_{\varepsilon}+\varphi_{\varepsilon}\right)\right)^{n} \leqslant \frac{C}{\varepsilon^{2 n}} C_{\varepsilon} \omega^{n},
$$

which gives $C_{\varepsilon} \geqslant c \varepsilon^{3 n}$.

\section{REFERENCES}

1. Błocki, Z. On the uniform estimate in the Calabi-Yau theorem, II, Sci. China Math. 54 (2011), no. 7, 1375-1377. MR2817572

2. Buchdahl, N. On compact Kähler surfaces, Ann. Inst. Fourier (Grenoble) 49 (1999), no. 1, 287-302. MR.1688136 (2000f:32029)

3. Cherrier, P. Équations de Monge-Ampère sur les variétés Hermitiennes compactes, Bull. Sc. Math (2) 111 (1987), 343-385. MR921559 (89d:58131)

4. Coman, D., Guedj, V. Quasiplurisubharmonic Green functions, J. Math. Pures Appl. (9) 92 (2009), no. 5, 456-475. MR2558420 (2011e:32044)

5. Demailly, J.-P. Champs magnétiques et inégalités de Morse pour la $d^{\prime \prime}$-cohomologie. Ann. Inst. Fourier (Grenoble) 35 (1985), 189-229. MR812325 (87d:58147)

6. Demailly, J.-P. A numerical criterion for very ample line bundles, J. Differential Geom. 37 (1993), no. 2, 323-374. MR1205448 (94d:14007)

7. Demailly, J.-P. Effective bounds for very ample line bundles, Invent. Math. 124 (1996), no. 1-3, 243-261. MR1369417 (97a:32035)

8. Demailly, J.-P. A converse to the Andreotti-Grauert theorem, Annales de la faculté des sciences de Toulouse, sér. 6, 20, no. S2 (2011), 123-135. DOI:10.5802/afst.1308 
9. Demailly, J.-P., Păun, M. Numerical characterization of the Kähler cone of a compact Kähler manifold, Ann. of Math. (2) 159 (2004), no. 3, 1247-1274. MR2113021 (2005i:32020)

10. Demailly, J.-P., Peternell, T. and Schneider, M. Compact complex manifolds with numerically effective tangent bundles, J. Algebraic Geom. 3 (1994), no. 2, 295-345. MR1257325 (95f:32037)

11. Dinew, S., Kołodziej, S. Pluripotential estimates on compact Hermitian manifolds, preprint, arXiv:0910.3937.

12. Gauduchon, P. Le théorème de l'excentricité nulle, C. R. Acad. Sci. Paris 285 (1977), 387-390. MR0470920 (57:10664)

13. Gill, M. Convergence of the parabolic complex Monge-Ampère equation on compact Hermitian manifolds, Analysis and Geometry 19 (2011), no. 2, 277-304. MR.2835881

14. Guan, B., Li, Q. Complex Monge-Ampère equations and totally real submanifolds, Adv. Math. 225 (2010), no. 3, 1185-1223. MR2673728 (2011g:32053)

15. Guedj, V., Zeriahi, A. Intrinsic capacities on compact Kähler manifolds, J. Geom. Anal. 15 (2005), no. 4, 607-639. MR2203165 (2006j:32041)

16. Hartshorne, R. Algebraic Geometry, Graduate Texts in Mathematics, No. 52. Springer-Verlag, New York-Heidelberg, 1977. MR0463157 (57:3116)

17. Hironaka, H. An example of a non-Kählerian complex-analytic deformation of Kählerian complex structures, Ann. of Math. (2) 75 (1962), 190-208. MR0139182 (25:2618)

18. Hörmander, L. An introduction to complex analysis in several variables, Van Nostrand, Princeton, N.J., 1973. MR0344507 (49:9246)

19. Ji, S. Image of analytic hypersurfaces. II, Math. Ann. 297 (1993), 693-706. MR:1245414 (95f:32030)

20. Kodaira, K. On the structure of compact complex analytic surfaces. I, Amer. J. Math. 86 (1964), 751-798. MR0187255(32:4708)

21. Lamari, A. Courants kählériens et surfaces compactes, Ann. Inst. Fourier (Grenoble) 49 (1999), no. 1, 263-285. MR1688140(2000d:32034)

22. Lazarsfeld, R. Positivity in Algebraic Geometry I, Springer, Berlin, 2004. MR2095471 (2005k:14001a)

23. Miyaoka, Y. Kähler metrics on elliptic surfaces, Proc. Japan Acad. 50 (1974), 533-536. MR 0460730 (57:723)

24. Siu, Y.T. Every K3 surface is Kähler, Invent. Math. 73 (1983), no. 1, 139-150. MR707352 $(84 \mathrm{j}: 32036)$

25. Siu, Y.-T. Effective very ampleness, Invent. Math. 124 (1996), no. 1-3, 563-571. MR1369428 (97a:32036)

26. Tian, G. On Kähler-Einstein metrics on certain Kähler manifolds with $c_{1}(M)>0$, Invent. Math. 89 (1987), 225-246. MR894378 (88e:53069)

27. Tian, G., Yau, S.-T. Kähler-Einstein metrics on complex surfaces with $C_{1}>0$. Comm. Math. Phys. 112 (1987), no. 1, 175-203. MR.904143 (88k:32070)

28. Tosatti, V., Weinkove, B. The complex Monge-Ampère equation on compact Hermitian manifolds, J. Amer. Math. Soc. 23 (2010), no. 4, 1187-1195. MR2669712

29. Tosatti, V., Weinkove, B. Estimates for the complex Monge-Ampère equation on Hermitian and balanced manifolds, Asian J. Math. 14 (2010), no. 1, 19-40. MR2726593 (2011h:32043)

30. Yau, S.-T. On the Ricci curvature of a compact Kähler manifold and the complex MongeAmpère equation. I, Comm. Pure Appl. Math. 31 (1978), no. 3, 339-411. MR480350 (81d:53045)

Department of Mathematics, Columbia University, 2990 Broadway, New York, New YORK 10027

E-mail address: tosatti@math.columbia.edu

Department of Mathematics, University of California San Diego, 9500 Gilman Drive, \#0112, La Jolla, CAlifornia 92093

E-mail address: weinkove@math.ucsd.edu 